\title{
Radiation-Hard Silicon Strip Sensors for the ATLAS Phase-2 Upgrade
}

\author{
Věra Latoňováa ${ }^{a, b, *}$, on behalf of the ATLAS Collaboration \\ ${ }^{a}$ Institute of Physics of the Czech Academy of Sciences, \\ Na Slovance 1999/2, 18221 Prague 8, Czech Republic \\ ${ }^{b}$ Institute of Particle and Nuclear Physics, Faculty of Mathematics and Physics, Charles University, \\ V Holešovičkách 2, 18000 Prague 8, Czech Republic \\ E-mail: vera.latonova@cern.ch
}

The ATLAS upgrade for HL-LHC operation includes the installation of an entirely new all-silicon Inner Tracker (ITk). The silicon strip region comprises $165 \mathrm{~m}^{2}$ of instrumented area, made possible by mass production of silicon strip sensors. This area is covered in a nearly hermetic way. Multiple sensor shapes are utilized: square sensors in the barrel part, and skewed trapezoidal sensors with curved edges to provide a continuous coverage of the disc surface in the endcap part of a detector. As a result, there are 8 different strip sensor types in the system. They all feature AC-coupled $\mathrm{n}^{+}$-in-p strips with polysilicon biasing, developed to withstand the total fluence of $1.6 \cdot 10^{15} \mathrm{n}_{\mathrm{eq}} / \mathrm{cm}^{2}$ and the total ionizing dose of $66 \mathrm{Mrad}$. Following many years of $\mathrm{R} \& \mathrm{D}$ and 4 prototype submissions and evaluations, the project transitioned into pre-production, where $5 \%$ of the total volume is produced in all 8 designs. In this contribution we will summarize the results obtained for the latest prototype sensors ATLAS17LS, a long-strip type sensor for the barrel part, the properties of which are expected to be comparable with the pre-production sensors.

40th International Conference on High Energy physics - ICHEP2020

July 28 - August 6, 2020

Prague, Czech Republic (virtual meeting)

\footnotetext{
${ }^{*}$ Speaker
} 


\section{Introduction}

The current Inner Detector (ID) was designed to work for 10 years at the peak luminosity of $10^{34} \mathrm{~cm}^{-2} \mathrm{~s}^{-1}$ [1]. During the Phase-II upgrade of the LHC into High Luminosity LHC (HL-LHC), the ID will be completely replaced by an all-silicon Inner Tracker (ITk) containing a new type of silicon sensors, which shall withstand the harsh radiation environment accompanying the total integrated luminosity of $4000 \mathrm{fb}^{-1}$ and provide sufficient granularity to keep detector occupancy comparable to ID even for the peak luminosity of $7.5 \cdot 10^{34} \mathrm{~cm}^{-2} \mathrm{~s}^{-1}[2,3]$. The outer part of the ITk will be covered by a new type of single-sided $\mathrm{n}^{+}$-in-p strip sensors manufactured on 6" p-type wafers with a thickness of $320 \mu \mathrm{m}$, which were developed for the total fluence of $1.6 \cdot 10^{15} \mathrm{n}_{\text {eq }} / \mathrm{cm}^{2}$ (including safety factor 1.5) [3]. The sensors' aluminum strips are AC-coupled to $\mathrm{n}^{+}$implants biased via polysilicon bias resistors. The barrel region of the strip part of the ITk will consist of two short strip (SS) layers followed by two long strip (LS) layers. The end-cap (EC) region will be made of twelve disks, six disks on each EC side, each of which will contain 6 different sensor designs (R0 - R5).

\section{Quality assessment program}

The quality assessment program consists of Quality Control (QC) and Quality Assurance (QA). QC verifies that the delivered sensors meet the specifications defined in the ITk Sensor Specifications Documents. It is performed on the main sensors, which will be used in the ITk detector, and includes tests on every sensor (visual inspection, IV, CV and metrology) and tests on sample sensors (leakage current stability, full strip tests and detailed strip tests). QA includes the tests performed on miniature sensors and test structures before irradiation, the irradiation itself and tests after irradiation, of such components sampled from every production batch to provide confidence that the requirements are fulfilled through the whole production [4]. A summary of the participating QC and QA testing sites can be found in the Table 1.

\begin{tabular}{c|c|c|c|c} 
Site & QC & Irrad. & QA (CCE) & QA (TC) \\
\hline Cambridge & $\mathrm{x}$ & & & \\
\hline QMUL & $\mathrm{x}$ & & & \\
\hline KEK/Tsukuba & $\mathrm{x}$ & $\mathrm{x}$ & $\mathrm{x}$ & $\mathrm{x}$ \\
\hline SCIPP & $\mathrm{x}$ & & & \\
\hline Vancouver cluster & $\mathrm{x}$ & & & \\
\hline Carleton & $\mathrm{x}$ & & & \\
\hline Prague & $\mathrm{x}$ & $\mathrm{x}$ & & $\mathrm{x}$ \\
\hline Birmingham & & $\mathrm{x}$ & $\mathrm{x}$ & $\mathrm{x}$ \\
\hline Ljubljana & & $\mathrm{x}$ & $\mathrm{x}$ & \\
\hline Toronto & & & $\mathrm{x}$ & $\mathrm{x}$ \\
\hline Valencia & & & $\mathrm{x}$ & \\
\hline CNM & & & & $\mathrm{x}$ \\
\hline
\end{tabular}

Table 1: List of the testing sites and their commitment in the QC and QA tests, where QA (CCE) and QA (TC) stand for the charge collection efficiency measurements on minis and the whole suite of tests on the ATLAS Test Chips, respectively.

\section{Effect of irradiation on measured sensor characteristics}

The irradiation by various particles causes changes in the sensor surface and bulk. The bulk damage is primarily caused by displacement of silicon atoms from their lattice sites, whereas the 
surface damage includes effects in the $\mathrm{SiO}_{2}$ layers and interface regions caused by ionization and formation of defects [5].

Due to the delay caused by Covid-19, we summarize the results from prototypes. The preproduction sensors are expected to show the same characteristics due to the identical fabrication process. The observable changes in the sensor characteristics caused by the bulk damage are shown to increase the leakage current linearly proportional to the fluence [5-7], to change the full depletion voltage with the fluence $[5,8,9]$ and to decrease the charge collection efficiency [5, 9]. The charges trapped in the $\mathrm{SiO}_{2}$ layers and the defects in the $\mathrm{Si}-\mathrm{SiO}_{2}$ interface are shown to be responsible for the increase of the inter-strip capacitance $[5,10]$, decrease of the inter-strip resistance [5, 7] and increase of the flat-band voltage [5]. In conclusion, the results described above clearly indicate that post-irradiation performance of the prototype sensors satisfies ATLAS requirements.

\section{Acknowledgments}

The research was supported and financed by the Ministry of Education, Youth and Sports of the Czech Republic coming from the project LM2015058 - Research infrastructure for experiments at CERN and by Charles University grant GAUK 942119.

\section{References}

[1] ATLAS Collaboration, The ATLAS Experiment at the CERN Large Hadron Collider, J. Instrum. 3 (2008) S08003.

[2] ATLAS Collaboration, Technical Design Report for the ATLAS Inner Tracker Strip Detector, Tech. Rep. CERN-LHCC-2017-005. ATLAS-TDR-025, CERN, Geneva (Apr, 2017).

[3] "RadiationBackgroundSimulationsStep3X." https://twiki . cern.ch/twiki/bin/ viewauth/Atlas/RadiationBackgroundSimulationsStep3X, Accessed on 07/24/2020.

[4] M. Ullán et al., Quality Assurance methodology for the ATLAS Inner Tracker strip sensor production, Nucl. Instrum. Methods. Phys. Res. A 981 (2020) 164521.

[5] F. Hartmann, Evolution of silicon sensor technology in particle physics; 2nd ed., Springer tracts in modern physics, Springer, Berlin (Dec, 2017).

[6] M. Moll, Displacement Damage in Silicon Detectors for High Energy Physics, IEEE Trans Nucl Sci (2018) 1.

[7] M. Mikestikova et al., Electrical characterization of surface properties of the ATLAS17LS sensors after neutron, proton and gamma irradiation, Nucl. Instrum. Methods. Phys. Res. A 983 (2020) 164456.

[8] V. Cindro et al., Measurement of the charge collection in irradiated miniature sensors for the upgrade of the ATLAS phase-II strip tracker, Nucl. Instrum. Methods. Phys. Res. A 924 (2019) 153.

[9] K. Hara et al., Charge collection study with the ATLAS ITk prototype silicon strip sensors ATLAS17LS, Nucl. Instrum. Methods. Phys. Res. A 983 (2020) 164422.

[10] M. Mikestikova et al., Study of surface properties of ATLAS12 strip sensors and their radiation resistance, Nucl. Instrum. Methods. Phys. Res. A 831 (2016) 197. 